\title{
Histoire de la psychiatrie et de la neurologie à Genève
}

Par Georges de Morsier

\section{Histoire de la psychiatrie à Genève}

$\mathrm{Au} \mathrm{XVIII}{ }^{\mathrm{e}}$ siècle, les aliénés étaient, à Genève comme ailleurs, entassés pêlemêle, hommes, femmes, enfants, dans des maisons «de force» et soumis à la brutalité des gardiens. A Genève, l'annexe de l'Hôpital Cantonal, situé alors au Bourg-de-Four (l'actuel Palais de Justice) portait le nom caractéristique de «Discipline». C'est actuellement la prison de Saint-Antoine, située à côté du Collège de Calvin.

Comme l'a écrit Mr Marc Cramer, c'est un médecin genevois, Abraham Joly (1748-1812), qui réalisa une réforme considérable en supprimant les chaînes et autres moyens barbares de coercition. Joly était Docteur-médecin de Montpellier en 1771, puis agrégé à la Faculté de Genève en 1774, mais il renonce à la pratique urbaine dès 1780 pour être nommé, l'année suivante, l'un des Directeurs de l'Hôpital, puis en 1787 «Hospitalier». L'Hôpital général du XVIII ${ }^{\mathrm{e}}$ siècle, comme la plupart de ses contemporains, rappelle plus un hospice ou un bureau de bienfaisance qu'un hôpital au sens moderne du mot... L' «Hospitalier», c'était la cheville ouvrière de l'Hôpital qu'il était chargé de gérer. On trouve dans les registres officiels de très grands éloges sur la gestion, le dévouement, la compétence, autant de Joly que de l'«Hospitalière», qui était Mme Joly. En 1788, l'Hôpital comptait une centaine de malades, dont une vingtaine d'aliénés. Les malheureux fous étaient non seulement enfermés à la «discipline» avec les condamnés de droit commun, mais la plupart du temps enchaînés.

Gautier, dans sa belle Histoire de la médecine à Genève (1905) écrit : «Précurseur de Pinel, Abraham Joly fit cesser à l'Hôpital de Genève l'emploi des chaînes et autres moyens barbares de coercition dont on se servait alors partout sous prétexte de soigner les aliénés. » Mais Gautier ne donne aucune référence. Or aucun registre officiel (Chambre de santé, Hôpital, même Registre du Conseil) ne fait une allusion quelconque à la délivrance des aliénés; on pouvait donc se demander si Gautier s'était laissé tromper par de vagues rumeurs. Mr Cramer a pu mettre la main sur une brochure publiée par Guillaume Firmin Moultou lors des études préliminaires pour la construction d'un nouvel hospice des aliénés en 1830 (asile des Vernets). G. F. Moultou, le fils de «l'ami de Rousseau» (1767-1832), a travaillé de nombreuses années à l'ancien Hôpital général avant d'en être nommé l'un des Directeurs. Il a donc connu Joly personnellement. Il écrit : «Genève n’était pas plus juste et plus humaine pour ses aliénés que les autres pays. Elle fut longtemps sous l'in- 
fluence et l'empire des préjugés qui maîtrisaient l'Europe ; l'établissement où ils étaient enfermés avait ses chaînes, ses chambres noires et nos fous étaient abandonnés aux seuls soins des infirmiers. Un hospitalier philanthrope, M. Joly, en introduisant de grandes améliorations dans notre Hôpital, s'occupa aussi de notre 'discipline'; il substitua le corset de force en toile aux chaînes et aux colliers de fer dont on se servait pour contenir les fous furieux. » Comme Joly fut Hospitalier de 1787 à 1792, il résulte que la réforme du traitement des aliénés dut être à peu près contemporaine de celle de Daquin à Chambéry et antérieure de plusieurs années à celle de Pinel qui fit si grand bruit (Bicêtre 1798, Salpêtrière 1801). Gautier avait donc raison de considérer Joly comme un précurseur de Pinel.

Il faut mentionner ici le Dr Charles Coindet (1796-1876), fils du Dr JeanFrançois Coindet, célèbre par ses travaux sur les propriétés thérapeutiques de l'iode. Docteur en Médecine d'Edimbourg, il revint à Genève où il donna une attention particulière à l'étude des maladies mentales. En 1835, il fut directeur de l'asile des Vernets puis, en 1858, directeur de la maison de santé de la Métairie à Nyon.

Un autre Genevois, Charles-Gaspard de la Rive réussit à faire sortir les aliénés de la Discipline et les transférer à la campagne. Il est né à Genève le 14 mars 1770. A peine âgé de 24 ans il fut condamné à mort par le tribunal révolutionnaire genevois en 1794. Il put s'évader de sa prison grâce aux sentiments de reconnaissance d'un geôlier à qui il avait rendu quelques services et se réfugia à Londres, puis à Edimbourg, où il consacra ses loisirs forcés à l'étude de l'anglais et de la médecine. Sa facilité pour le travail et ses rares aptitudes lui valurent d'être nommé très promptement président de la Société royale de médecine de cette ville et lui permirent de passer brillamment ses examens de doctorat. Puis il visita les principaux asiles d'aliénés d'Angleterre, en étudia minutieusement le fonctionnement et revint à Genève en 1799, où il fut nommé professeur de physique à l'Académie. Outre son activité psychiatrique Gaspard de la Rive a été un grand physicien qui, avec son fils Auguste, a fait des travaux très importants pour la découverte de l'électro-magnétisme ${ }^{1}$.

En 1802 il est chargé du soin des aliénés, alors placés à la Discipline. «Dans ces locaux défectueux, insalubres et exigus, il s'applique à donner aux aliénés tous les soins désirables avec intelligence et bonté, et en supprimant complètement les fauteuils de force, les tourniquets, les bains de surprise et autres cruelles inventions d'une époque où l'on pensait généralement qu'il fallait se rendre maître des fous par la violence et l'intimidation. Il réclame, aidé par son ami le juriste Etienne Dumont, la création d'un asile qui soit le résultat de toutes les lumières acquises 
sur cet objet. » Chose curieuse, c'est sous la pression de la nécessité - l'épidémie de choléra qui s'abattit sur l'Europe en 1832 - qu'on dut débarrasser d'urgence la Discipline pour y mettre les cholériques et transporter les aliénés à la campagne, dans une grande maison louée pour la circonstance et située à Corsier, à $8 \mathrm{~km}$ de Genève. Les aliénés y séjournèrent de 1832 à 1838. Après leur départ, cette maison devint la «fruiterie» de Corsier.

A quelque chose malheur était bon, car jamais les aliénés, qui bénéficiaient grandement d'être à l'air et à la campagne, ne réintégrèrent la Discipline. De la Rive s'employa activement à l'éviter. «A aucun prix, écrit-il, il ne faut permettre la réintégration de ces malheureux à la Discipline. Cette mesure serait fatale pour nos malades qui changeraient un bon air, de vastes cours, de grands jardins et de jolies chambres contre leur triste prison, leur cour étroite avec ses hautes murailles froides, humides, malsaines. Cette mesure serait aussi odieuse pour le public. Rappelons le spectacle déchirant de ces femmes, de ces hommes, demi-nus, s'attachant aux barreaux de leur prison et provoquant par leurs cris de désespoir, leurs clameurs féroces, les promeneurs, les passants, les étrangers indignés de ce spectacle, et nos jeunes écoliers sortant de leur classe qui venaient par groupes se démoraliser à ce déplorable passe-temps.»

Gaspard de la Rive mourut le 18 mars 1834, mais l'idée qu'il avait défendue pendant 32 ans d'activité auprès des aliénés avec autant de dévouement que de compétence fut reprise par son successeur le Dr Jean-Charles Coindet et triompha peu après par l'inauguration de l'asile des Vernets, à la queue d'Arve, dans lequel les aliénés furent transportés le $1^{\text {er }}$ juin 1838, venant de Corsier, au nombre de 57 ( 27 hommes, 28 femmes et 2 enfants).

Dans L'Académie et l'Université de Genève au XIX siècle (Annexes), nous voyons que la loi sur l'Instruction publique du 5 juin 1886 qui a remplacé, en ce qui concerne la Faculté de médecine, celle du 24 septembre 1873, prévoit entre autres la création de cours de Clinique psychiatrique (Art. 130): Il est confié au docteur Olivet un cours annuel de deux heures par semaine. «L'enseignement, à la fois théorique et pratique, se donne à l'Hospice des Vernaies. A chaque séance, le cours est immédiatement suivi de l'application ou de la démonstration sur le malade... Le personnel de la Clinique psychiatrique, pour ce qui concerne l'Université, comprend: le professeur; un assistant, qui est en même temps médecin en second de l'établissement et le personnel subalterne nécessaire» (p. 273).

Le successeur du Dr Olivet fut Johannès Martin (1851-1939), né à Versoix le 25 août 1851. Après avoir fréquenté les collèges d'Evian et de Fribourg, il se rend à l'Université de Munich pour passer son année de sciences naturelles et se préparer au premier propédeutique; il travaille ensuite, à la Faculté de Médecine de 
Berne, les disciplines nécessaires au second propédeutique. Il part ensuite pour Vienne où il se perfectionne en otorhino-laryngologie sous la direction de Politzer ; il fréquente encore les grands hôpitaux de Lyon et acquiert ainsi une culture médicale très solide. En 1876 il rentre à Genève et il est nommé médecin-assistant à l'Hospice des Vernets; il s'oriente alors vers la psychiatrie. Pour parfaire son éducation dans cette discipline, il occupe un poste d'assistant au Burghölzli, l'Asile cantonal des aliénés du canton de Zurich. En 1880 il obtint son diplôme fédéral de médecin à Berne. Peu de temps après, il est nommé médecin-directeur de l'Asile de Marsens à Fribourg et pendant quatre ans, de 1880 à 1884, il se dépensera pour transformer et réorganiser cette maison. En 1884, il rentre momentanément à Genève pour s'adonner à la pratique médicale; mais la psychiatrie le hante et il repart pour Vienne suivre les cours et les cliniques mentales, en particulier celles de Meynert. De retour à Genève, il reprend sa vie de praticien, en l'entrecoupant chaque année par des séjours à Paris. Dans cette ville, il élabore sous la direction de Jules Déjerine un mémoire sur l'Atrophie du nerf optique et sa valeur dans la sclérose des cordons postérieurs de la moelle. Ce mémoire présenté comme thèse lui vaudra le grade de docteur en médecine de l'Université de Berne (1899).

Il devient alors médecin-adjoint de l'Hospice des Vernets et supplée le professeur Marc-André Olivet. Après la retraite de ce dernier il est nommé médecindirecteur du vieil asile le 6 novembre 1891. Il continue à assurer bénévolement les cours de psychiatrie; le $1^{\text {er }}$ décembre 1896, enfin, il est nommé professeur ordinaire. Johannès Martin se mit sans retard au travail pour procéder à la réorganisation administrative et médicale de la maison des Vernets, afin de pouvoir améliorer le sort des malades et leur assurer des soins appropriés. Il s'efforça de changer l'atmosphère de l'asile pour en faire un hôpital. La construction d'un nouvel asile, à Bel-Air près de Chêne, ayant été décidée, J. Martin prit une part active aux études nécessaires et suivit de près les travaux de construction. Les crédits furent dépassés, la politique s'en mêla et J.Martin, découragé, préféra donner sa démission, le 10 octobre 1899, en alléguant son état de santé.

Quelques mois après, il acceptait la direction de la Métairie, près de Nyon, maison de santé privée, et il occupa ce poste près de six années. En 1906, le professeur J.Martin rentre dans la vie privée et vient s'établir à Genève. Il accepte quelques fonctions officielles: membre du conseil de surveillance psychiatrique, expertises devant les tribunaux, etc. Avec les années, devenu moins ingambe, il renonça à toutes les fonctions officielles dont il avait accepté la charge et il se retira dans sa maison de Veyrier. C'est là qu'il décéda, après une courte maladie le 12 janvier 1939, à l'âge de 88 ans. 
A cette biographie, le Professeur Charles Ladame, dans un article nécrologique, ajoute différentes notes qu'il nous faut reproduire : «Son grand âge n'avait en rien affecté ni sa vive intelligence, ni sa curiosité toujours en éveil, moins encore sa grande bonté et sa sensibilité si affinée... L'œuvre essentielle de Johannès Martin remonte à plus d'un demi-siècle... Cette œuvre est faite d'initiatives dans tous les domaines de l'économie et de la vie d'une telle institution, initiatives pour la plupart qui, pour l'époque, étaient assurément celles d'un pionnier de la psychiatrie la plus moderne. Il nous plaît d'avoir été chargé de rendre un juste hommage à la mémoire de notre regretté confrère, d'évoquer cette figure aussi noble que modeste et de marquer les traits caractéristiques de son œuvre qui constitue une des belles pages de l'histoire du régime des aliénés à Genève. Mais nous avons dû reconstituer cette histoire de toutes pièces car, homme d'action avant tout, Johannès Martin n'a laissé que peu d'écrits. Les archives du Conseil d'Etat, le Mémorial du Grand Conseil, les rapports annuels des asiles publics d'aliénés dont il fut le médecin-directeur, les thèses de doctorat en médecine qu'il inspira à ses élèves nous ont procuré heureusement les documents officiels... Cependant la note intime qui parachève à souhait notre portrait émane des entretiens nombreux qu'il nous a été donné d'avoir eus avec lui ces années dernières... Ses propos, marqués au coin d'un grand bon sens et d'une intelligence aussi avertie que bien équilibrée, étaient ceux d'un homme qui a beaucoup vu, côtoyé de près les misères des hommes et dont le cœur est resté inaltérablement bon, ouvert et compréhensif... En ce temps-là, la situation de l'asile des Vernets, qui était déplorable, allait s'aggravant de jour en jour. L'encombrement des services était à son comble, le désordre et la gabegie régnaient partout en maîtres. Le directeur laïque, sans aucune compétence en ces matières spéciales, était débordé, ne savait vraiment plus où donner de la tête. Le médecin en chef, subordonné au directeur en quelque sorte, ne résidait pas à l'asile. Il s'y rendait pour de courtes apparitions, tout au plus deux fois par semaine. Il connaissait peu les malades qu'il voyait à peine un instant... Nous avons eu l'avantage, il y a quelques années, en compagnie du docteur G. de Morsier, de parcourir les ruines lamentables des Vernets. Non sans satisfaction, Martin put nous montrer, de visu, comment il s'y était pris, il y avait plus de 50 ans, pour créer des salles de surveillance continue, équipées d'appareils électriques pour le contrôle des rondes et des veilles... Il instaura le traitement par le lit ou 'clinothérapie', innovation importante entre toutes... L'asile des Vernets, un modèle du genre lors de sa création en 1838, allait être désaffecté... Les années 1892 à 1899 furent pour J. Martin huit années fécondes, mais d'un labeur dur et constant. Elles furent couronnées de succès aussi bien par la réussite de la réorganisation des Vernets que par la conception et l'édification de Bel-Air 
et par l'équipement intérieur du nouvel établissement ou hôpital psychiatrique dont Genève allait sous peu être dotée... Il expose les éléments de ces principes et la loi genevoise révisée en 1895 dans les Annales médico-psychologiques de Paris. Mandons qu'il avait été nommé membre associé de cette compagnie... Le professeur Martin a fait partie de bien des corps savants. Aux deux déjà cités, ajoutons la Société médicale de Genève, la Société médico-psychologique de Genève, à l'existence éphémère de laquelle il a contribué en acceptant la présidence dès sa fondation pendant deux ans. C'est alors, avec le Dr G. de Morsier, que fut élaborée la deuxième révision de la loi genevoise sur le régime des aliénés de 1838 , révisée en 1895. Cet avant-projet a fait l'objet de nombreuses études et discussions au sein de notre Société. Ce n'est toutefois qu'en 1935 que ce projet, sorti des cartons du Département de l'Hygiène, fut mis à l'étude et accepté par le pouvoir législatif en 1936... Et à nous, ses confrères qui l'avons aimé et fort apprécié, sa mort nous laisse le chagrin amer d'avoir perdu un guide, un ami sûr et éclairé, un confrère aussi modeste que probe qui a honoré notre profession selon la mode ancienne. »

Le successeur de Johannès Martin fut Rodolphe Weber (1866-1937), qui entra en fonction en janvier 1900. D'origine saint-galloise, il est né à Oberuzwil. Il fait ses études secondaires à Neuchâtel où son père, médecin lui-même, était venu s'établir. Après avoir étudié la médecine à Zurich, Berne et Munich, il obtint le grade de docteur à Bâle, en 1891, avec une thèse intitulée: Über 25 Fälle von Geistesstörung nach Kopfverletzung, qui était déjà un travail de psychiatrie. Il s'installe ensuite à La Neuveville, où il pratique quelque temps. Il entre ensuite dans le service de l'Asile d'aliénés de la Waldau à Berne; il continue sa formation par un stage à la Clinique psychiatrique de Bâle et enfin à l'Asile de Münsingen du canton de Berne. Il fut alors nommé à Genève professeur ordinaire, le 9 janvier 1900, et directeur du futur asile de Bel-Air. De lourdes tâches l'attendaient, celle d'achever l'aménagement du nouvel établissement pour maladies mentales, celle d'achever d'y transférer les malades de l'ancien Asile des Vernets et celle de créer les laboratoires nécessaires à l'examen anatomo-pathologique des cerveaux atteints d'altérations provoquant des troubles psychiques. R. Weber s'attela à ces tâches et réussit à les mener à bien.

Rodolphe Weber ne manifesta pas l'ambition de publier ses observations; son intérêt était tout entier voué à ses malades, au diagnostic et au traitement de leurs maladies. Il a donc peu écrit et son œuvre est avant tout d'un médecin qui a inspiré une estime sincère et chaleureuse. Il aimait à descendre le soir dans le silence du laboratoire pour s'y livrer à la préparation technique des pièces récoltées lors des autopsies. Ces précieux documents lui servaient à illustrer son enseigne- 
ment aux étudiants; il cherchait la base de ses conceptions bien plus dans ses études personnelles que dans la littérature. Ses leçons avaient une note individuelle qui en faisait le charme.

Si Rodolphe Weber a peu écrit lui-même, sa modestie était trop grande, il mettait en revanche libéralement ses observations anatomiques et cliniques au service de ses assistants et de ses élèves; de nombreuses thèses ont été publiées sous sa direction. Il avait exercé avec distinction les fonctions de doyen de la Faculté de Médecine de 1912 à 1915 et celles de recteur de l'Université de 1922 à 1924. C'est immédiatement après qu'il prit sa retraite et il fut nommé honoraire le 18 novembre 1924. Sa carrière universitaire était terminée, celle de psychiatre continua, car Rodolphe Weber se plaça à la tête d'une clinique privée, installée dans la maison historique de Sismondi. Il y exerça, pendant treize ans, son activité bienfaisante. Il est décédé le 24 avril 1937.

Max Azkanazy, qui était alors professeur de pathologie, était devenu l'ami de Rodolphe Weber. Dans une notice nécrologique, il note ceci : «Le trait caractéristique de cette nature était la modestie. Dans ma longue carrière académique, je ne trouve personne qui, à cet égard, pourrait lui être comparé... Sa connaissance de six langues lui servit non seulement dans sa clientèle privée, mais dans ses goûts littéraires; il ne se lassait pas de lire avec pénétration, dans la langue originale, les écrivains représentant l'essence littéraire de ces six cultures. Si sa modestie est restée le trait prépondérant de sa personnalité, c'est parce qu'il comparait le savoir humain à la grandeur de la Création, à la beauté insaisissable de la nature... Il a aimé cette nature, attiré surtout par les beautés de son pays. Silencieuse, fidèle et sincère comme l'affection qu'il avait pour son pays, telle était son amitié humaine... Que ses amis conservent un souvenir reconnaissant de Rodolphe Weber et cherchent à s'inspirer des beaux principes qui l'ont guidé dans son existence féconde.»

Charles Ladame (1871-1949) était neuchâtelois d'origine; il est né au Locle le 19 juillet 1871. Il fit ses études de médecine à Genève, à Zurich et à Lausanne ; i] les termina dans cette dernière ville, en 1903 , en présentant une thèse de doctorat décrivant Un nouveau parasite du cancer, le corpuscule de Feinberg. Charles Ladame était attiré par le laboratoire et il s'est initié aux recherches histologiques normales et pathologiques, en occupant à Lausanne divers postes d'assistant; il garda le goût de la recherche scientifique au cours de sa carrière. Fils de Paul Ladame, Charles Ladame fut très tôt attiré par la neurologie et la psychiatrie. Au lendemain de sa thèse, il entra comme premier assistant à la clinique psychiatrique de Cery à Lausanne. Puis, de 1908 à 1918, il fut le médecin adjoint de l'Asile-Clinique de Bel-Air, dirigé par le professeur Rodolphe Weber. En 1919, Ch. Ladame devint 
privat-docent, sur présentation d'un travail d'habilitation intitulé : Histologie des maladies mentales. En qualité de privat-docent, il enseigna avec distinction la psychiatrie théorique durant plusieurs années. De cette époque date une série de travaux importants se rapportant tous à l'étude anatomo-pathologique du cerveau. Il convient de relever son rapport sur Les bases anatomiques des psychoses (1909), son mémoire sur La structure architectonique de l'écorce cérébrale (1911); sa revue sur L'encéphale, son évolution anatomique et fonctionnelle et sa pathologie. Le souci de chercher la lésion organique correspondant aux troubles psychiques observés en clinique est resté sa préoccupation majeure, et, en 1936, il publiait encore, en collaboration avec le docteur F. Morel, une Contribution à la topographie des lésions histologiques du cerveau sénile.

Les circonstances éloignèrent momentanément de Genève Charles Ladame, qui exerça de 1919 à 1924 les fonctions de médecin suppléant du directeur à l'Asile psychiatrique de la Rosegg dans le canton de Soleure.

A ce moment, la chaire de psychiatrie devint vacante à Genève, à la suite de la démission du professeur Rodolphe Weber. Charles Ladame fut désigné, le 14 novembre 1924, comme professeur ordinaire des maladies mentales et directeur de l'Asile-clinique de Bel-Air. Il remplit ces fonctions durant quatorze ans, avec une conscience scrupuleuse et une bienveillance inlassable. Comme professeur, son enseignement était captivant et ses leçons cliniques toujours orientées par le souci de retrouver le soubassement organique des troubles psychiques qu'il décrivait sans se laisser influencer par toutes les théories qui avaient éclos.

Absorbé par la lourde tâche de médecin et de directeur d'une clinique qu'il fallut agrandir à plusieurs reprises, désireux d'améliorer continuellement les conditions hospitalières et le traitement des malades qui lui étaient confiés, le professeur Charles Ladame a dû restreindre son activité scientifique pour l'orienter vers les problèmes pratiques qui s'imposaient à lui. En 1929, il publia le fruit de ses expériences de médecin directeur dans un mémoire intitulé: Réformes et rationalisation dans le régime de l'Asile-clinique de Bel-Air; ce mémoire fut traduit en allemand et en italien.

Devenu très dur d'oreille, atteint de cataracte, Charles Ladame décida de prendre sa retraite pour raison de santé. Il fut nommé professeur honoraire le 28 juin 1938. Opéré de la cataracte, quelques mois plus tard, il a raconté dans une note biographique ses impressions avant, pendant et après l'opération qu'il avait subie en 1940, et il exprime sa joie et sa reconnaissance d'avoir recouvré une vision suffisante pour pouvoir de nouveau lire, écrire et travailler les documents qu'il avait réunis au cours de sa carrière. La mort vint soudaine; le professeur Charles Ladame décédait le 28 octobre 1949. 
C'est Ferdinand Morel (1888-1957) qui succéda au Prof. Charlez Ladame comme professeur de psychiatrie et directeur de la Clinique psychiatrique de BeJAir. Je pense pouvoir reproduire ici l'hommage que j'ai prononcé à la Faculté de Médecine de Genève le 23 octobre 1957 : «Il n’entrerait pas dans le cadre de cet hommage à notre ami disparu, de retracer, même brièvement, l'œuvre scientifique considérable de celui qui, par l'ampleur de ses connaissances, la sûreté de son jugement et la rigueur de sa méthode, fut un véritable humaniste. J'aimerai faire ressortir ici seulement deux des qualités de cet esprit exceptionnel: son extrême probité scientifique, tout au long de sa carrière de philosophe, de psychologue, puis de psychiatre et le rayonnement de sa personnalité morale.

Bachelier en théologie à Neuchâte] dès 1911, mais imbu de la méthode cartésienne, Morel n'a pas trouvé dans la transcendance ni dans la dogmatique à satisfaire son besoin passionné de connaissance et de compréhension de la vie, même religieuse. Cherchant sa voie, il entre en contact avec le professeur Théodore Flournoy, parti lui de la médecine pour devenir un maître de la philosophie et de la psychologie religieuse pour laquelle il avait posé, dès 1890 , le principe de la méthode: l'exclusion de la transcendance. Pendant l'hiver 1913-14, Morel suit le cours de Flournoy faisant connaître à Genève la doctrine que Freud édifiait à Vienne depuis dix ans sur l'enseignement qu'il avait reçu à la Salpêtrière. On sait que Freud a édifié sa doctrine sur l'Hystérie de Charcot, qui n'a pas survécu à son auteur... Théologien devenu philosophe et psychologue, Morel cherche alors à expliquer les phénomènes religieux très complexes que présentent les mystiques par les concepts que Freud, Jung et Bleuler d'une part, et Pierre Janet de l'autre, venaient d'introduire dans l'explication des phénomènes psychologiques morbides et qui suscitaient alors de grands espoirs. L'érotisme déchaîné de certains mystiques et la récente doctrine de Freud, qui mettait l'instinct sexuel à la base de toute la psychologie normale et pathologique, expliquent aisément pourquoi Morel a pensé pouvoir serrer de plus près le vieux problème du mysticisme, pierre d'achoppement de la théologie et de la philosophie. De là est née sa thèse de doctorat en philosophie: Essai sur l'introversion mystique. Etude psychologique de Pseudo-Denys l'Aréopagite et de quelques autres cas de mysticisme, volume de 336 pages paru en 1918 et dédié à Théodore Flournoy... Il tente d'expliquer les phénomènes mystiques par les concepts des psychanalystes : introversion, autisme, narcissisme, symbolisme, fixation érotique à la mère ou au père, etc. Mais Morel est visiblement déçu du peu de résultats qu'il a obtenu et il conclut modestement: «Il n'est donc pas possible encore de dire grand'chose de précis, concernant l'introversion ou l'extraversion en général.» 
Cependant, en étudiant minutieusement l'histoire des mystiques, Morel constate que tous présentent des symptômes morbides rencontrés habituellement chez les malades mentaux: hallucinations visuelles, auditives, sensations génitales imposées, impression de lévitation, angoisses, phobies, obsessions, impulsion, automatismes, paralysies, double personnalité, délires de persécution, d'auto-accusation, de possession diabolique et aussi des perversions sexuelles... Il s'aperçoit qu'il serait tout de même plus facile d'étudier les nombreux introvertis malades à l'Asile de Bel-Air que les anciens mystiques morts depuis des siècles. Il obtient alors du professeur Rodolphe Weber la permission de converser avec des malades de l'Asile. Mais il sait que ces introvertis ont tous des maladies du cerveau, qu'il faudrait connaître et comprendre. C'est alors que le docteur Charles Ladame, qui était alors médecin adjoint de l'Asile de Bel-Air, lui dit : «Pourquoi n'étudieriezvous pas la médecine?»

Rompant alors avec ses études passées, reniant sa thèse de philosophie, renonçant au cours de privat-docent qu'il donnait à la Faculté des lettres depuis 1919 , sourd aux appels les plus flatteurs que lui adressaient philosophes, psychologues et psychanalystes et qui lui assuraient un avenir intellectuel facile, voyant que seules d'autres méthodes pourraient l'amener à la connaissance des phénomènes qu'il avait observés, Morel s'inscrit à la Faculté de Médecine. Il a 33 ans. Ses maîtres seront alors Amédée Weber pour la neuro-histologie, André Thomas et Clérambault pour la clinique neurologique et psychiatrique... Cette extrême probité, Morel en a donné une nouvelle preuve, vingt ans après sa thèse de philosophie, lors de sa leçon inaugurale à la chaire de Clinique psychiatrique en 1938. Au lieu d'exalter la valeur exceptionnelle et l'importance de la discipline qu'il allait enseigner, comme c'est toujours le cas en pareille circonstance, jugeant impitoyablement l'échec de l'Essai sur l'introversion mystique, Morel a dit ceci : «Il n'existe pas, que je sache, une méthode propre à la psychiatrie. L'histoire de cette discipline nous montre bien une floraison de méthodes accessoires dont beaucoup ont été sans lendemain. Elles n'ont joué qu'un rôle accessoire parce qu'elles ne permettaient pas de sortir du champ clos de la description. Or, la description pour la description n'est encore qu'une activité littéraire au sein de la médecine. » L'excellence des résultats obtenus par Morel dans son œuvre scientifique a montré le bien fondé de ce «Discours de la méthode» en psychiatrie.

Grâce à sa clairvoyance et à sa probité, Morel a empêché que des centaines de malades de Genève aient été gravement mutilés. Alors que les «lobotomies», ou sections chirurgicales des lobes frontaux du cerveau faisaient fureur en Amérique, puis dans le monde entier, il s'est opposé énergiquement à cette intervention. Avec raison il s'indignait de voir des psychiatres, qui ne s'étaient jamais intéressés 
à l'anatomie ni à la physiologie du cerveau, faire pratiquer cette mutilation qui altère définitivement la personnalité, sur des malades sans défense. Il restait indifférent aux sarcasmes de ceux qui l'accusaient de ne pas suivre la mode du jour. L'abandon de ces opérations mutilantes montre à quel point il avait raison et quelle reconnaissance nous lui devons... Il a eu sur ses malades qu'il connaissait tous personnellement, sur ses collaborateurs, ses élèves et sur tous ceux qui l'ont approché, l'influence la plus bienfaisante. Il s'est ingénié à donner à ses malades une ambiance toujours plus agréable, plus confortable, plus esthétique même et les résultats qu'il a obtenus sont très remarquables.

Morel a publié 71 travaux, tous très importants. Il a résumé son œuvre dans un très beau livre, paru en 1947 : Introduction à la psychiatrie neurologique.

En 1929, avec F. Morel, nous avons tenté de montrer que la «schizophrénie», concept exposé avec beaucoup de talent par Eugen Bleuler (1916), n'est pas incurable, car de nombreux patients étiquetés «schizophrènes» guérissent, mais qu'ils sont handicapés toute leur vie par ce diagnostic d'incurabilité. D'autre part, les phénomènes élémentaires des psychoses, comme Baillarger, Séglas (1890), enfin et surtout G.G. de Clérambault (1920) l'ont montré, sont les hallucinations verbales, verbomotrices, visuelles, olfactives, gustatives, l'écho de la pensée, l'énonciation des actes, les intuitions, les convictions. C'est secondairement que les différents délires sont construits par les patients à partir de ces mécanismes élémentaires.

Après la mort de Ferdinand Morel, c'est le Professeur Juan de Ajuriaguerra qui a été appelé à prendre sa succession. 
${ }^{1}$ M. Cramer et G. de Morsier, L'importance des expériences faites à Genève par Gaspard et Auguste De la Rive pour la découverte de l'électro-magnétisme, in : Gesnerus 28 (1971), p. 234-245.

\section{Bibliographie}

Askanazy, Max, Rodolphe Weber (1866-1937).

Bleuler, Eugen, Lehrbuch der Psychiatrie, 2. Auflage, Berlin (Julius Springer) 1918.

Bujard, Eugène, La Faculté de Médecine de 1914 à 1956. L'Université de Genève de 1914 à 1956. Annexes. Genève (Georg \& Cie), p.218, 257, 260, 272.

Cramer, Marc (Musée d'Histoire des Sciences, Genève), Un précurseur de Pinel : Abraham Joly. Actes de la Société helvétique des sciences naturelles, 1974, p.165-167.

Eternod, Auguste C.F., La Faculté de Médecine de 1876 à 1896. L'Académie et l'Université de Genève au XIX ${ }^{e}$ siècle. Annexes. Genève (Georg \& Cie) 1959, p.242 et 272.

Gautier, Léon, La médecine à Genève jusqu'à la fin du dix-huitième siècle (avec onze portraits), Genève (J. Jullien, Georg \& Cie) 1906.

Ladame, Charles, Johannès Martin (1851-1939). Revue médicale de la Suisse romande, LIX $^{\mathrm{e}}$ année, $\mathrm{n}^{\mathrm{o}} 8,25$ juin 1939, p. 493-505.

Ladame, Paul-Louis, Un médecin aliéniste précurseur: Gaspard de la Rive (1770-1834). Congrès des médecins aliénistes et neurologistes de France et des pays de langue française, $\mathrm{XVII}^{\mathrm{e}}$ session tenue a Genève et à Lausanne du $1^{\mathrm{er}}$ au 7 août 1907 (13 pages et un portrait).

Morel, Ferdinand, Notice nécrologique sur Charles Ladame (1871-1949). Revue médicale de la Suisse romande, $69^{\mathrm{e}}$ année, 1949, p. 919.

Morel, Ferdinand, Introduction à la psychiatrie neurologique, Paris (Masson) et Lausanne (F. Roth \& Cie) 1947 (298 p., 57 fig.).

Morsier, Georges de, L'Hygiène mentale à Genève. Conférence faite à l'assemblée générale annuelle de la Société de Prophylaxie mentale et de Patronage, le 29 mars 1918. Genève (Société générale d'Imprimerie) 1928. (Une brochure de 19 pages avec un portrait.)

Morsier, Georges de, Ferdinand Morel (1888-1957). Revue médicale de la Suisse romande, LXXIX $^{\mathrm{e}}$ année, $\mathrm{n}^{\circ} 1$, janvier 1958, p. 44-47.

Morsier, Georges de, et Morel, F., Critique de la notion de schizophrénie. Annales MédicoPsychologiques, $\mathrm{n}^{\circ}$ 5, décembre 1929. Communication faite à la Séance commune de la Société suisse de Psychiatrie et de la Société des Psychiatres du Sud-Ouest de l'Allemagne, Bâle, octobre 1929. 


\section{Histoire de la neurologie à Genève}

L'enseignement de la neurologie a tout d'abord été fait par les professeurs de Clinique médicale. Un nombre important de travaux concernant les maladies du système nerveux ont été élaborés dans ces cliniques. Dans le travail qu'il a consacré à son maître Léon Revilliod (1835-1918), professeur de Clinique médicale à Genève pendant 23 ans, Edouard Claparède, qui a été son assistant, a donné la liste des travaux concernant le système nerveux faits par Revilliod ou ses élèves. Ils sont au nombre de 18. Le plus connu est le «signe de l'orbiculaire de la paupière» (1892). C'est sous la direction de Revilliod que Claparède a fait sa thèse : Du sens musculaire, à propos de quelques cas d'Hémiataxie posthémiplégique (1897).

Le successeur de Léon Revilliod, le professeur Louis Bard (1857-1930) a consacré aux maladies du système nerveux 33 travaux, dont les plus connus sont ses recherches sur le nystagmus.

Le professeur Maurice Roch, qui a succédé en 1920 à Louis Bard, s'est toujours beaucoup intéressé aux maladies du système nerveux. Ainsi il a consacré 43 de ses remarquables Dialogues cliniques à des maladies du système nerveux.

Le Docteur Paul-Louis Ladame (1842-1919) était membre correspondant étranger de l'Académie de Médecine de Paris. Son père était professeur de chimie et de physique à l'Académie de Neuchâtel. Il a fait ses études de médecine aux Universités de Zurich, Wurzbourg, Berne et Paris. C'est en 1865, alors qu'il était assistant à l'Hôpital Pourtalès de Neuchâtel qu'il a publié une remarquable thèse Symptomatologie und Diagnostik der Hirngeschwülste, forte de 265 pages. C'était le travail le plus complet paru jusqu'alors sur ce sujet, encore mal connu des cliniciens.

Son activité dans le domaine de la médecine sociale a été considérable et, là aussi, il a été un précurseur. Il fut chargé par le gouvernement neuchâtelois d'étudier les orphelinats suisses et étrangers, la protection et l'assistance aux enfants abandonnés, anormaux, orphelins et illégitimes.

En 1884 il fait des stages dans les cliniques d'électrologie, de neurologie et de psychiatrie à Paris et à Berlin. C'est en 1884 qu'il vient se fixer à Genève. Il y a professé comme privat-docent pendant 24 ans à la Faculté de médecine, et parfois à la Faculté de droit, sur différents sujets de neurologie, de psychiatrie médicolégale, d'anthropologie criminelle, d'histologie du système nerveux. Son travail sur l'aphasie, fait en collaboration avec son ami le professeur von Monakow, de Zurich, est très connu. En outre, il a fait d'importants travaux d'archives, en particulier le Procès criminel de la dernière sorcière brûlée à Genève le 6 avril 1652 (Michée Chaudron), paru dans la Bibliothèque diabolique, et Une séquestration 
arbitraire dans la Maison des Aliénés à Genève au XVIII ${ }^{e}$ siècle. Procédure démenée contre Jean-Louis Damoisel (1758-1779), publiée dans le Bulletin de l'Institut national genevois d'août 1922.

Edouard Long (1868-1929) est né à Genève le 7 juillet 1868. Il était le fils du Dr Ernest Long qui avait été interne des hôpitaux de Paris, puis directeur de «La Métairie», près de Nyon, enfin médecin de l'Hôpital cantonal de Genève. Edouard Long commence ses études scientifiques puis médicales à l'Université de Genève. Après avoir réussi son examen propédeutique fédéral, il part pour Paris et y achève sa formation médicale. En 1893 il réussit le concours de l'internat et, l'année suivante, il entre dans le service du Docteur Déjerine à Paris. Ceci détermina toute sa carrière: il se vouera à l'anatomie et à la pathologie des centres nerveux. Sa thèse de doctorat est consacrée aux Voies de la sensibilité générale. Etude anatomo-clinique (1899), travail considérable, fort de 277 pages, dans lequel il démontre que, contrairement à Charcot, les voies de la sensibilité générale ont un relais dans le thalamus.

Peu après Edouard Long revient à Genève où, intéressé par l'enseignement, il subit les épreuves de privat-docent et présente une thèse d'habilitation : Contribution à l'étude des scléroses de la moelle épinière, sclérose en plaques et syphilis médullaire. Cependant, l'amitié qui le lie au professeur J. Déjerine et les ressources scientifiques que lui offre le laboratoire de la Salpêtrière l'attirent chaque année à Paris durant les mois d'hiver. A Genève, Long fréquente le service de la Clinique médicale où, grâce à la libéralité de ses directeurs, les professeurs Louis Bard, puis Maurice Roch, il peut étudier les cas nerveux, les présenter à son cours et créer un laboratoire. Ses leçons, bien préparées, claires et objectives, attirent les étudiants; il les illustre grâce à la collection de coupes et de pièces anatomiques qu'il a créée patiemment; il projette des films cinématographiques qui complètent ses présentations cliniques. A côté de son enseignement, Long organise des visites régulières qui groupent autour de lui les médecins neurologues. La première guerre mondiale interrompt cette activité. A Bourg-en-Bresse il est à la tête d'un service neurologique de plus en plus chargé ; il dirige, dans l'ancien couvent de Veyrier, l'hôpital où sont recueillis et soignés les évacués du nord de la France. La guerre finie, il reprend ses occupations à Genève.

La Faculté de Médecine propose alors la création d'une chaire de Neurologie qui lui serait donnée pour consacrer vingt ans d'enseignement bénévole. Le 5 août 1919, il est nommé professeur extraordinaire de Neuro-pathologie. Sa vaste érudition, sa science toujours au point, sa rigoureuse probité scientifique, son accueil toujours cordial firent de lui le consultant préféré des médecins qui lui assuraient une autorité méritée. 
Edouard Long a laissé de nombreux travaux scientifiques qu'il a écrits seul ou souvent en collaboration avec sa femme, Madame Long-Landry; presque tous sont consacrés à la neurologie et trop nombreux pour être analysés dans cette brève notice. Ont peut citer Les plaies des nerfs dans les blessures de guerre (1917), La sclérose en plaques et son étiologie, L'atrophie de la mcelle épinière dans la sclérose en plaques, Le diagnostic différentiel de la sclérose en plaques et des compressions médullaires.

Ses nombreux et remarquables travaux valurent à Edouard Long de multiples distinctions. Il fut nommé membre correspondant de la Société de psychiatrie à Paris; lauréat de l'Académie des Sciences (prix Martin-Damonvelt) en 1901. Il présida le Congrès des aliénistes et neurologistes de France et des pays de langue française, tenu à Genève en 1926.

Depuis quelque temps sa santé donnait lieu à des inquiétudes, lorsque le 2 août 1920 il fut terrassé brusquement par une attaque et expira en quelques minutes.

Dans L'Université de Genève de 1914 à 1956 (Annexes), le professeur Eugène Bujard écrit (page 205) : La chaire de Neuropathologie resta vacante plusieurs années malgré le désir manifesté à plusieurs reprises par la Faculté. En décembre 1933, la Société académique offrit d'assumer pour trois ans les frais de cet enseignement. A la suite de cette offre, le Dr Georges de Morsier, privat-docent de neurologie et de psychiatrie, fut désigné comme chargé de cours de neurologie le 31 janvier 1934, avec une heure d'enseignement par semaine durant toute l'année. Un malentendu suspendit cette charge en 1936, mais le 25 octobre 1938, le Dr de Morsier fut réinstallé dans sa charge. Il fut nommé professeur extraordinaire de neurologie le 6 décembre 1941. Le titre du cours inscrit au programme devint celui de Clinique de neurologie et de neuropathologie avec une heure de cours pendant un semestre; toutefois, comme cela avait été prévu antérieurement par la Faculté, la neurologie ne jouit pas d'un service hospitalier indépendant, mais elle reste attachée à la Clinique de médecine. La même année le professeur G. de Morsier organise un laboratoire attaché à son service.

En 1942 le professeur G. de Morsier demande que son enseignement soit de deux heures par semaine en été, ce à quoi la Faculté acquiesce. De plus il informe celleci qu'il se propose d'organiser, en été aussi, des travaux de recherche de neuropathologie.

En 1945 un laboratoire de psychologie appliquée à la clinique est créé dans le service de neurologie et la direction en est confiée au professeur André Rey, professeur associé à la Faculté des sciences ${ }^{1}$.

A plusieurs reprises, la Faculté discute de l'opportunité de rendre le service neurologique indépendant. Ce n'est qu'en 1952 que cette proposition trouve un 
accueil favorable, mais la réalisation est renvoyée jusqu'au moment où le professeur M. Roch, directeur de la Clinique médicale, aura pris sa retraite.

En 1953 le professeur Georges Bickel, nouveau titulaire de la chaire de médecine, consent à détacher de celle-ci un service de neuropathologie de 56 lits. Ce service dispose du personnel suivant : un médecin adjoint bénévole, un premier assistant fonctionnant comme chef de clinique, deux assistants et un chef de laboratoire de psychologie clinique (le professeur André Rey) ${ }^{2}$.

\section{Notes}

${ }^{1}$ C'est le professeur Edouard Claparède qui a fondé ce laboratoire et créé le terme de "psychologie clinique» en 1938. Après la mort de Claparède (1940), le professeur André Rey a pris la direction du laboratoire de psychologie clinique et lui a donné une grande extension.

${ }^{2}$ J'ai été nommé professeur ordinaire et directeur de la Clinique universitaire de Neurologie en 1960 et directeur de la policlinique de Neurologie en 1962.

\section{Bibliographie}

Bickel, Georges, Le professeur Maurice Roch (1878-1967). Médecine et Hygiène 25, $\mathrm{n}^{\circ} 722$, 22 mars 1967.

Bujard, Eugène, L'Université de Genève de 1914 à 1956. Annexes. Genève (Georg \& Cie) 1959, p. 205-206.

Claparède, Edouard, Vingt-trois ans de professorat : M. Léon Revilliod. La Suisse universitaire, janvier 1900.

Cottin, M ${ }^{\text {elle }}$ le Docteur, L'œeuvre scientifique du professeur Louis Bard (1857-1930). (Un volume de 210 pages avec un portrait et la liste des 378 publications de Louis Bard.)

Maillard, Hector, Le Professeur Edouard Long (1868-1929). Revue médicale de la Suisse romande, $89^{\mathrm{e}}$ année, $\mathrm{n}^{\mathrm{o}} 11,25$ septembre 1929 , p. 717-719.

Mozer, Jean-Jacques, Maurice Roch (1878-1967), Médecin et Humaniste genevois, Genève 1973.

Naville, François, Le docteur Paul-Louis Ladame, de Genève (15 juin 1842-21 octobre 1919). Archives suisses de Neurologie et de Psychiatrie 5, fasc. 2, 2 février 1919.

Naville, François, Notice nécrologique sur le professeur Edouard Long. Archives suisses de Neurologie et de Psychiatrie 25 (1929), p.165-170.

Roch, Maurice ; Cramer, Alexandre ; Naville, François ; Maillard, Hector ; Roussy, Gustave; Babonneix Dr; André-Thomas Dr; Naville, François, Discours prononcés aux obsèques du Docteur Edouard Long le 10 août 1929. Imprimé à Paris le 15 octobre 1930 sur les presses de l'Imprimerie Lahure. (Brochure de 66 pages avec un portrait, suivie de la liste des 83 publications d'Edouard Long.) 


\section{Summary}

\section{History of Psychiatry at Geneva}

During the 18th century the insane were incarcerated in the "Discipline" (at present St. Antoine prison), where they were manhandled. In 1787 Abraham Joly took their chains off. His reform antidates thus the one carried through by Pinel at Paris by several years. In 1802 Gaspard de la Rive transfered the insane to the country side (Corsier). From there he brought them to the Asylum of Vernet, constructed in 1838 and directed by Johannès Martin. Later they came to the psychiatric clinic of Bel-Air which was successively directed by Rodolphe Weber, Charles Ladame, Ferdinand Morel and Juan de Ajuriaguerra.

\section{History of Neurology at Geneva}

At Geneva the teaching of neurology was first in the hands of the professors of internal medicine: Léon Revilliod, Louis Bard, Paul-Louis Ladame, Maurice Roch. Edouard Long, the disciple of Jules Déjerine at Paris, wrote in a remarquable thesis on "the pathways of general sensibility" that these pathways have a relais in the thalamus, denied by Charcot. After his return to Geneva he became professor of neuropathology.

Prof. Dr. Georges de Morsier 1 , promenade du Pin 1204 Genève 